\title{
Epidermal Growth Factor Receptor Mutations and Their Prognostic Value with Carcinoembryonic Antigen in Pathological T1 Lung Adenocarcinoma
}

\author{
Wang-Yu Zhu $\mathbb{D}^{1,2,3}$ Hai-Feng Li, ${ }^{4}$ Ke-Xin Fang, ${ }^{1}$ Bing-Jie Zhang, ${ }^{2,3}$ Shi-Quan Zhou, \\ Yong-Kui Zhang, ${ }^{2,3}$ Han-Bo Le $\mathbb{D}^{2,3}$ and Xiao-Fei Hu ${ }^{2}{ }^{2}$ \\ ${ }^{1}$ Cellular and Molecular Biology Laboratory, Zhoushan Hospital of Wenzhou Medical University, Zhoushan, Zhejiang, China \\ ${ }^{2}$ Lung Cancer Research Centre, Zhoushan Hospital of Wenzhou Medical University, Zhoushan, Zhejiang, China \\ ${ }^{3}$ Department of Cardio-Thoracic Surgery, Zhoushan Hospital of Wenzhou Medical University, Zhoushan, Zhejiang, China \\ ${ }^{4}$ Department of Respiratory Medicine, Zhoushan Hospital of Wenzhou Medical University, Zhoushan, Zhejiang, China
}

Correspondence should be addressed to Wang-Yu Zhu; zhuwangyu24@sina.cn,Han-Bo Le; zslehanbo@163.com, and Xiao-Fei Hu; 1097824003@qq.com

Received 25 August 2017; Revised 24 January 2018; Accepted 4 February 2018; Published 24 April 2018

Academic Editor: Michele Malaguarnera

Copyright $\odot 2018$ Wang-Yu Zhu et al. This is an open access article distributed under the Creative Commons Attribution License, which permits unrestricted use, distribution, and reproduction in any medium, provided the original work is properly cited.

\begin{abstract}
Aims. The prognostic value of epidermal growth factor receptor (EGFR) mutations in the context of serum carcinoembryonic antigen levels remains controversial in T1 lung adenocarcinoma. Methods. Clinical and pathological characteristics, preoperational carcinoembryonic antigen levels, EGFR mutations, and disease-free and overall survival were analysed retrospectively in 573 pathological T1 patients in East China. Results. EGFR mutations were detected in 220 of 573 patients (38.4\%). Patients with serum carcinoembryonic antigen levels $\geq 2.12 \mathrm{ng} / \mathrm{mL}$ had worse disease-free $(P<0.001)$ and overall survival $(P<0.001)$ than had others, although survival was comparable between patients with and without EGFR mutations. However, patients with exon 21 mutations in EGFR had significantly better overall survival than had patients with exon 19 mutations $(P=0.016)$, although disease-free survival was comparable $(P=0.424)$. Among patients with serum carcinoembryonic antigen levels $\geq 2.12 \mathrm{ng} / \mathrm{mL}$, disease-free $(P=0.019)$ and overall survival $(P<0.001)$ was also better than that in those with exon 21 mutations. Finally, the exon 19 deletion was found to be an independent predictor of unfavourable overall survival $(P=0.037)$. Conclusions. EGFR mutations were associated with preoperational serum carcinoembryonic antigen levels $\geq 2.12 \mathrm{ng} / \mathrm{mL}$. In patients with levels above this threshold, those with the exon 19 deletion have less favourable prognosis than have those with the exon 21 mutation.
\end{abstract}

\section{Introduction}

Lung adenocarcinoma is the most prevalent subtype of non-small cell lung cancer, and the 5-year overall survival remains poor [1-3]. Lung adenocarcinoma may arise from an accumulation of genetic mutations, of which those in epidermal growth factor receptor (EGFR) are some of the most important and are associated with tumour progression, proliferation, and survival [4]. EGFR mutations are the most common genetic lesions in adenocarcinoma but are very rare in squamous cell carcinoma [5]. However, the median progression-free survival in squamous cell carcinoma patients treated with EGFR tyrosine kinase inhibitors is worse than that in adenocarcinoma patients with EGFR mutations [6]. Thus, EGFR mutations may be predictive of the therapeutic response to such inhibitors [7]. Similarly, non-small cell lung cancer patients with mutated EGFR also have higher median disease-free survival and improved overall survival [8-10]. However, the predictive value of EGFR mutations in patients with pathological T1 lung adenocarcinoma is still unclear. Strikingly, deletion of EGFR exon 19 and a point mutation in exon 21 account for up to $90 \%$ of EGFR mutations in the clinic and correspond to two distinct tumour subtypes with different clinical characteristics and 
TABLE 1: Clinical and pathologic features of patients with lung adenocarcinoma with a maximum diameter of $3.0 \mathrm{~cm}$ or less and stratified by presence and absence of EGFR mutations. Where appropriate, data are $n(\%)$.

\begin{tabular}{|c|c|c|c|c|}
\hline Characteristics & All $(n=573)$ & EGFR mutated $(n=220)$ & EGFR wild type $(n=353)$ & $P$ \\
\hline Age, mean y (range) & $57.0 \pm 10.0(26-84)$ & $60.5 \pm 10.0(28-84)$ & $55.0 \pm 10.5(26-77)$ & $<0.001^{* a}$ \\
\hline \multicolumn{5}{|l|}{ Sex } \\
\hline Male & $175(30.5)$ & $69(39.4)$ & $106(60.6)$ & \multirow[t]{2}{*}{$0.780^{\mathrm{b}}$} \\
\hline Female & $398(69.5)$ & $151(37.9)$ & $247(62.1)$ & \\
\hline \multicolumn{5}{|l|}{ Smoking } \\
\hline Nonsmoker & $468(81.7)$ & $184(39.3)$ & $284(60.7)$ & \multirow[t]{2}{*}{$0.375^{\mathrm{b}}$} \\
\hline Current/former & $105(18.3)$ & $36(34.3)$ & $69(65.7)$ & \\
\hline \multicolumn{5}{|l|}{ CEA } \\
\hline$<2.12 \mathrm{IU} / \mathrm{mL}$ & $378(66.0)$ & $133(35.2)$ & $245(64.8)$ & \multirow[t]{2}{*}{$0.030^{* b}$} \\
\hline$\geq 2.12 \mathrm{IU} / \mathrm{mL}$ & $195(34.0)$ & $87(44.6)$ & $108(55.4)$ & \\
\hline \multicolumn{5}{|l|}{ Type of surgery } \\
\hline Lobectomy & $392(68.4)$ & $156(39.8)$ & $236(60.2)$ & \multirow[t]{2}{*}{$0.356^{\mathrm{b}}$} \\
\hline Limited & $181(31.6)$ & $64(35.4)$ & $117(64.6)$ & \\
\hline \multicolumn{5}{|l|}{ Tumour size } \\
\hline pT1a & $305(53.2)$ & $76(24.9)$ & $229(75.1)$ & \multirow[t]{3}{*}{$<0.001^{* b}$} \\
\hline pT1b & $197(34.4)$ & $103(52.3)$ & $94(47.7)$ & \\
\hline pT1c & $71(12.4)$ & $41(57.7)$ & $30(42.3)$ & \\
\hline \multicolumn{5}{|l|}{ Histology } \\
\hline AIS & $170(29.7)$ & $34(20.0)$ & $136(80.0)$ & \multirow[t]{3}{*}{$<0.001^{* b}$} \\
\hline MIA & $167(29.1)$ & $57(34.1)$ & $110(65.9)$ & \\
\hline IAC & $236(41.2)$ & $129(54.7)$ & $107(45.3)$ & \\
\hline \multicolumn{5}{|l|}{ IAC subtype $(n=236)$} \\
\hline Lepidic & $53(22.5)$ & $30(56.6)$ & $23(43.4)$ & \multirow[t]{5}{*}{$0.041^{* \mathrm{c}}$} \\
\hline Acinar & $65(27.5)$ & $40(62.5)$ & $25(37.5)$ & \\
\hline Papillary & $105(44.5)$ & $57(54.3)$ & $48(45.7)$ & \\
\hline Solid & $9(3.8)$ & $2(22.2)$ & $7(77.8)$ & \\
\hline Mucinous variant & $4(1.7)$ & 0 & $4(100.0)$ & \\
\hline \multicolumn{5}{|l|}{ Pleural invasion } \\
\hline No & $528(92.1)$ & $195(36.9)$ & $333(63.1)$ & \multirow[t]{2}{*}{$0.016^{* b}$} \\
\hline Yes & $45(7.9)$ & $25(55.6)$ & $20(44.4)$ & \\
\hline \multicolumn{5}{|l|}{ Lymph nodes } \\
\hline pN0 & $546(95.3)$ & $204(37.4)$ & $342(62.6)$ & \multirow[t]{2}{*}{$0.026^{* \mathrm{~b}}$} \\
\hline $\mathrm{pN} 1$ or 2 & $27(4.7)$ & $16(59.3)$ & $11(40.7)$ & \\
\hline \multicolumn{5}{|l|}{ Pathological stage } \\
\hline 0 & $170(29.7)$ & $34(20.0)$ & $136(80.0)$ & \multirow[t]{4}{*}{$<0.001^{*}$} \\
\hline IA & $339(59.2)$ & $149(44.0)$ & $190(56.0)$ & \\
\hline IB & $37(6.4)$ & $21(56.8)$ & $16(43.2)$ & \\
\hline IIA, IIB, IIIA & $27(4.7)$ & $16(59.3)$ & $11(40.7)$ & \\
\hline
\end{tabular}

CEA: carcinoembryonic antigen; AIS: adenocarcinoma in situ; MIA: minimally invasive adenocarcinoma; IAC: invasive adenocarcinoma. ${ }^{*} P<0.05$ by ${ }^{\mathrm{a}} t$-test,

${ }^{\mathrm{b}}$ Pearson's chi-squared test, and ' Fisher's exact test.

response to EGFR tyrosine kinase inhibitors $[11,12]$. Hence, the prognostic role of EGFR mutations in T1 lung adenocarcinoma is probably well-defined.

In contrast, carcinoembryonic antigen has been used as a biomarker of prognosis and therapeutic efficacy in non-small cell lung cancer. Notably, Cai [13] reported that carcinoembryonic antigen levels gradually increase with the rate of EGFR mutations. Moreover, carcinoembryonic antigen levels were reported to be independently prognostic in lung adenocarcinoma patients without EGFR mutations [14]. Indeed, we also found that lung adenocarcinoma patients with carcinoembryonic antigen levels above $2.12 \mathrm{ng} / \mathrm{mL}$ have a poor prognosis [15]. Nevertheless, other surveys indicated that carcinoembryonic antigen levels are normal in most patients with early-stage lung cancer. Hence, the purpose of this study was to investigate EGFR mutations in the context 
of carcinoembryonic antigen levels and to assess the prognostic value of such mutations in patients with pathological T1 lung adenocarcinoma.

\section{Methods}

2.1. Patients. Patients who underwent surgical resection for pathological T1 adenocarcinoma of the lung $(N=573)$ were enrolled retrospectively at Zhoushan Hospital, Zhejiang, China, from July 2011 through March 2016. Histological subtypes were assigned by two pathologists, in accordance with World Health Organization classification and new criteria from the International Association for the Study of Lung Cancer, American Thoracic Society, and European Respiratory Society [16]. The staging of all patients with lung cancer was redefined according to the proposed 8th edition of lung cancer classification [17-19]. The maximum diameter of the resected lesion was measured by a pathologist. Patients were also genotyped for EGFR mutations. Clinicopathological features including age, sex, comorbidities, smoking history, lymphatic vessel invasion, vascular vessel invasion, pleural invasion, tumour maximum diameter, tumour stage, tumour histologic subtype, and preoperative serum carcinoembryonic antigen levels were analysed systematically. Patients with resected tumours greater than $3 \mathrm{~cm}$ in maximum diameter were excluded. Patients with incomplete records and follow-up data were also excluded, along with patients who died within 30 days after surgery. Patients were monitored over time, using computed tomography (CT) to assess recurrence. Overall survival was calculated as the period from surgical resection to end of follow-up, which was considered to be the time of death, or at the final follow-up of surviving patients. Disease-free survival was calculated as the period between surgery and initial detection of recurrence and metastasis. The study was approved by the Ethical Review Committee of the Zhoushan Municipal Government, and written informed consent was obtained from subjects or their families.

\subsection{Genomic DNA Extraction and EGFR Genotyping.} Resected tumours were fixed with $10 \%$ formalin, embedded in paraffin, and sectioned at $10 \mu \mathrm{m}$. Genomic DNA was extracted from five sections using a QIAamp DNA FFPE Tissue Kit (QIAGEN, Hilden, Germany). DNA concentration and purity were assessed on a Quawell Q3000 spectrophotometer (Quawell Technology, Sunnyvale, CA, USA). EGFR was genotyped on a 7500 Real-Time PCR System (ABI, Foster City, CA, USA) using an amplification refractory mutation system (Yuanqi Diagnostics, Shanghai, China), following the manufacturer's instructions.

2.3. Statistical Analysis. Data were analysed in GraphPad Prism 5.0 (GraphPad Software Inc., San Diego, CA) and SPSS 17.0 (SPSS Institute, Chicago, IL, USA). Pearson's chi-squared test or Fisher's exact test $(t<1$ or $n<40)$ was performed to compare differences between categorical groups. The Gaussian distribution was examined according to the Kolmogorov-Smirnov test. Then, the Mann-Whitney $U$ test [data shown as the median $\left(P_{25}, P_{75}\right)$ ] or unpaired

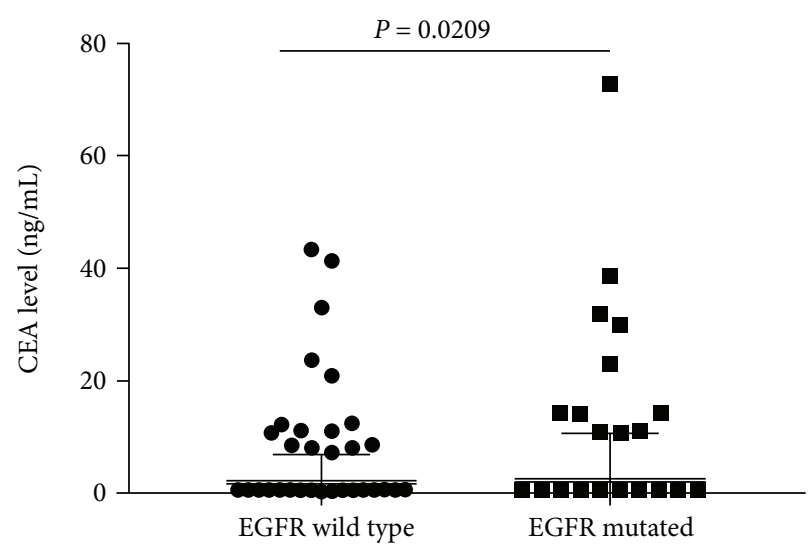

FIgURE 1: Box and whisker plot of preoperational CEA levels between EGFR mutated $(n=220)$ and wild-type $(n=353)$ lung carcinoma patients $[1.83(1.22,2.91)$ versus $1.61(1.05,2.54)$, $P=0.0209]$.

$t$-test (data shown as the mean $\pm \mathrm{SD}$ ) was used to analyse the difference in CEA level between EGFR mutated and wild-type patients. Disease-free and overall survival was evaluated by Kaplan-Meier curves. Multivariate analysis was performed using Cox's proportional hazards regression model for all prognostic factors with univariate $P<0.05$. All statistical tests were two-sided, with $P<0.05$ considered significant.

\section{Results}

3.1. Relationship between EGFR Mutations and Clinicopathological Features. Clinical and pathological characteristics are summarized in Table 1 for 573 patients in eastern Chinese islands who had lung adenocarcinoma with a maximum diameter of $3.0 \mathrm{~cm}$ or less. Of these patients, 220 were found postsurgery to harbour EGFR mutations (38.4\%), consisting of G719X in exon $18(n=6,1.0 \%)$, exon 19 deletion $(n=64,11.2 \%)$, exon 20 insertion $(n=1,0.2 \%)$, L858R and/or L861Q in exon 21 ( $n=145,25.3 \%)$, and combined mutations in exons 18 and $19(n=1,0.2 \%)$, exons 20 and $21(n=1,0.2 \%)$, and exons 19 and $21(n=2,0.3 \%)$. The CEA level was higher in EGFR mutated patients than in the wild-type patients $[1.83(1.22,2.91)$ versus 1.61 (1.05, 2.54), $P=0.0209$, Figure 1]. Mutations were more likely to occur in patients with carcinoembryonic antigen $\geq 2.12 \mathrm{ng} / \mathrm{mL}(P=0.030)$, a threshold identified in our previous survey [15]. Mutations were also associated with tumour size pT1b and pT1c $(P<0.001)$, pleural invasion $(P=0.016)$, histology $(P<0.001)$, lymphatic metastasis $(P=0.026)$, and stage $(P<0.001)$. All other clinical features were comparable between patients with and without EGFR mutations (Table 1).

Among patients with carcinoembryonic antigen levels $<2.12 \mathrm{ng} / \mathrm{mL}$ (Table 2), EGFR mutations were associated with age $(P<0.001)$, tumour size $(P<0.001)$, histology $(P<0.001)$, and stage $(P<0.001)$. In contrast, EGFR mutations in patients with carcinoembryonic antigen 
TABLE 2: Clinical and pathological features of T1 lung adenocarcinoma patients stratified by carcinoembryonic antigen (CEA) levels. Where appropriate, data are $n(\%)$.

\begin{tabular}{|c|c|c|c|c|c|c|}
\hline \multirow{2}{*}{ Characteristics } & \multicolumn{2}{|c|}{$\mathrm{CEA}<2.12 \mathrm{IU} / \mathrm{mL}(n=378)$} & \multirow{2}{*}{$P$} & \multicolumn{2}{|c|}{$\mathrm{CEA} \geq 2.12 \mathrm{IU} / \mathrm{mL}(n=195)$} & \multirow{2}{*}{$P$} \\
\hline & EGFR mutated & EGFR wild type & & EGFR mutated & EGFR wild type & \\
\hline Age, mean y (range) & $58.4 \pm 10.2(26-83)$ & $52.9 \pm 10.5(26-83)$ & $<0.001^{* a}$ & $63.7 \pm 8.3(38-84)$ & $59.8 \pm 8.6(38-84)$ & $0.002^{* a}$ \\
\hline \multicolumn{7}{|l|}{ Sex } \\
\hline Male & $37(16.3)$ & $190(83.7)$ & $0.260^{\mathrm{b}}$ & $55(49.1)$ & $57(50.9)$ & $0.143^{\mathrm{b}}$ \\
\hline Female & $96(63.6)$ & $55(36.4)$ & & $32(38.6)$ & $51(61.4)$ & \\
\hline \multicolumn{7}{|l|}{ Smoking } \\
\hline Nonsmoker & $115(35.0)$ & $214(65.0)$ & $0.873^{\mathrm{b}}$ & $69(49.6)$ & $70(50.4)$ & $0.038^{\mathrm{b}}$ \\
\hline Current/former & $18(36.7)$ & $31(63.3)$ & & $18(32.1)$ & $38(67.9)$ & \\
\hline \multicolumn{7}{|l|}{ Type of surgery } \\
\hline Lobectomy & $89(36.3)$ & $156(63.7)$ & $0.574^{\mathrm{b}}$ & $67(45.6)$ & $80(54.4)$ & $0.738^{\mathrm{b}}$ \\
\hline Limited & $44(33.1)$ & $89(66.9)$ & & $20(41.7)$ & $28(58.3)$ & \\
\hline \multicolumn{7}{|l|}{ Tumour size } \\
\hline pT1a & $58(23.9)$ & $185(76.1)$ & $<0.001^{* \mathrm{~b}}$ & $18(29.0)$ & $44(80.0)$ & $0.010^{* \mathrm{~b}}$ \\
\hline pT1b & $60(53.6)$ & $52(46.4)$ & & $43(50.6)$ & $42(49.4)$ & \\
\hline pT1c & $15(65.2)$ & $8(34.8)$ & & $26(54.2)$ & $22(45.8)$ & \\
\hline \multicolumn{7}{|l|}{ Histology } \\
\hline AIS & $29(21.5)$ & $106(78.5)$ & $<0.001^{* \mathrm{~b}}$ & $5(14.3)$ & $30(85.7)$ & $<0.001^{* b}$ \\
\hline MIA & $35(28.2)$ & $89(71.8)$ & & $22(51.2)$ & $21(48.8)$ & \\
\hline IAC & $69(58.0)$ & $50(42.0)$ & & $60(51.3)$ & $57(48.7)$ & \\
\hline \multicolumn{7}{|l|}{ IAC subtype $(n=236)$} \\
\hline Lepidic & $20(60.6)$ & $13(39.4)$ & $0.344^{\mathrm{c}}$ & $10(50.0)$ & $10(50.0)$ & $0.123^{\mathrm{c}}$ \\
\hline Acinar & $19(41.3)$ & $27(58.7)$ & & $21(56.8)$ & $16(43.2)$ & \\
\hline Papillary & $30(76.9)$ & $9(23.1)$ & & $27(56.3)$ & $21(43.7)$ & \\
\hline Solid & & & & $2(22.2)$ & $7(77.8)$ & \\
\hline Mucinous variant & 0 & $1(100.0)$ & & 0 & $3(100.0)$ & \\
\hline \multicolumn{7}{|l|}{ Pleural invasion } \\
\hline No & $124(34.3)$ & $238(65.7)$ & $0.105^{\mathrm{b}}$ & $71(42.8)$ & $95(57.2)$ & $0.231^{\mathrm{b}}$ \\
\hline Yes & $9(56.3)$ & $7(43.7)$ & & $16(55.2)$ & $13(44.8)$ & \\
\hline \multicolumn{7}{|l|}{ Lymph nodes } \\
\hline pNo & $130(34.8)$ & $244(65.2)$ & $0.127^{\mathrm{c}}$ & $74(43.0)$ & $98(57.0)$ & $0.267^{\mathrm{b}}$ \\
\hline pN1 or 2 & $3(75.0)$ & $1(25.0)$ & & $13(56.5)$ & $10(43.5)$ & \\
\hline \multicolumn{7}{|l|}{ Pathological stage } \\
\hline 0 & $29(21.5)$ & $106(78.5)$ & $<0.001^{* \mathrm{c}}$ & $5(14.3)$ & $30(85.7)$ & $0.001^{* \mathrm{c}}$ \\
\hline IA & $93(41.5)$ & $131(58.5)$ & & $56(48.7)$ & $59(51.3)$ & \\
\hline IB & $8(53.3)$ & $7(46.7)$ & & $13(59.1)$ & $9(40.9)$ & \\
\hline IIA, IIB, IIIA & $3(75.0)$ & $1(25.0)$ & & $13(56.5)$ & $10(43.5)$ & \\
\hline
\end{tabular}

${ }^{*} P<0.05$ by ${ }^{\mathrm{a}}$ t-test, ${ }^{\mathrm{b}}$ Pearson's chi-squared test, and ${ }^{\mathrm{c}}$ Fisher's exact test.

levels $\geq 2.12 \mathrm{ng} / \mathrm{mL}$ were associated with age $(P=0.002)$, nonsmokers $(P=0.038)$, tumour size $(P=0.010)$, histology $(P<0.001)$, and stage $(P<0.001$, Table 2$)$.

The clinical features of patients with an exon 19 deletion or an exon 21 point mutation are listed in Table 3. Exon 21 point mutations were more common in patients with the lepidic predominant invasive adenocarcinoma (IAC) subtype $(P=0.022)$. However, the frequency of mutations was comparable among other IAC subtypes and was not associated with other clinical and pathological features (Table 3 ).
3.2. Disease-Free and Overall Survival for Pathological T1 Lung Adenocarcinoma. The mean follow-up time was 27.7 months, with a median of 25 months and range of 2.5-68 months. Disease-free and overall survival was comparable between patients with and without EGFR mutations. However, patients with exon 21 mutations had significantly better overall survival than had patients with exon 19 mutations $(P=0.016)$, although diseasefree survival was comparable $(P=0.424$, Figure 2$)$. In addition, patients with serum carcinoembryonic antigen levels $\geq 2.12 \mathrm{IU} / \mathrm{mL}$ exhibited worse disease-free $(P<0.0001)$ 
TABLE 3: Clinical and pathological features of T1 lung adenocarcinoma patients with EGFR exon 19 deletion or exon 21 point mutations. Where appropriate, data are $n(\%)$.

\begin{tabular}{|c|c|c|c|}
\hline Characteristics & Exon $19(n=64)$ & Exon $21(n=145)$ & $P$ \\
\hline Age, mean y (range) & $59.7 \pm 10.7(30-80)$ & $61.2 \pm 9.4(28-84)$ & $0.324^{\mathrm{a}}$ \\
\hline \multicolumn{4}{|l|}{ Sex } \\
\hline Male & $24(35.8)$ & $43(64.2)$ & \multirow[t]{2}{*}{$0.263^{\mathrm{b}}$} \\
\hline Female & $40(28.2)$ & $102(71.8)$ & \\
\hline \multicolumn{4}{|l|}{ Smoking } \\
\hline Nonsmoker & $50(28.6)$ & $125(71.4)$ & \multirow[t]{2}{*}{$0.158^{\mathrm{b}}$} \\
\hline Current/former & $14(41.2)$ & $20(58.8)$ & \\
\hline \multicolumn{4}{|l|}{ CEA } \\
\hline$<2.12 \mathrm{IU} / \mathrm{mL}$ & $42(33.9)$ & $82(66.1)$ & \multirow[t]{2}{*}{$0.218^{\mathrm{b}}$} \\
\hline$\geq 2.12 \mathrm{IU} / \mathrm{mL}$ & $22(25.9)$ & $63(74.1)$ & \\
\hline \multicolumn{4}{|l|}{ Type of surgery } \\
\hline Lobectomy & $48(31.6)$ & $104(68.4)$ & \multirow[t]{2}{*}{$0.737^{\mathrm{b}}$} \\
\hline Limited & $16(28.1)$ & $41(71.9)$ & \\
\hline \multicolumn{4}{|l|}{ Tumour size } \\
\hline pT1a & $16(24.2)$ & $50(75.8)$ & \multirow[t]{3}{*}{$0.231^{\mathrm{b}}$} \\
\hline pT1b & $32(31.1)$ & $71(68.9)$ & \\
\hline pT1c & $16(40.0)$ & $24(60.0)$ & \\
\hline \multicolumn{4}{|l|}{ Histology } \\
\hline AIS & $6(20.0)$ & $24(80.0)$ & \multirow[t]{3}{*}{$0.267^{\mathrm{b}}$} \\
\hline MIA & $15(27.8)$ & $39(72.2)$ & \\
\hline IAC & $43(34.4)$ & $82(65.6)$ & \\
\hline \multicolumn{4}{|l|}{ IAC subtype $(n=125)$} \\
\hline Lepidic & $3(10.7)$ & $25(89.3)$ & \multirow[t]{4}{*}{$0.022^{* c}$} \\
\hline Acinar & $16(40.0)$ & $24(60.0)$ & \\
\hline Papillary & $23(41.8)$ & $32(58.2)$ & \\
\hline Solid & $1(50.0)$ & $1(50.0)$ & \\
\hline \multicolumn{4}{|l|}{ Pleural invasion } \\
\hline No & $54(29.3)$ & $130(70.7)$ & \multirow[t]{2}{*}{$0.278^{\mathrm{b}}$} \\
\hline Yes & $10(40.0)$ & $15(60.0)$ & \\
\hline \multicolumn{4}{|l|}{ Lymph nodes } \\
\hline pNo & $59(30.6)$ & $134(69.4)$ & \multirow[t]{2}{*}{$0.955^{\mathrm{b}}$} \\
\hline $\mathrm{pN} 1$ or 2 & $5(31.3)$ & $11(68.7)$ & \\
\hline \multicolumn{4}{|l|}{ Pathological stage } \\
\hline 0 & $6(20.7)$ & $24(79.3)$ & \multirow[t]{4}{*}{$0.216^{\mathrm{b}}$} \\
\hline IA & $43(30.1)$ & $99(69.9)$ & \\
\hline IB & $10(47.6)$ & $11(52.4)$ & \\
\hline IIA, IIB, IIIA & $5(31.3)$ & $11(68.7)$ & \\
\hline
\end{tabular}

CEA: carcinoembryonic antigen; AIS: adenocarcinoma in situ; MIA: minimally invasive adenocarcinoma; IAC: invasive adenocarcinoma. ${ }^{*} P<0.05$ by ${ }^{\mathrm{a}} t$-test, ${ }^{\mathrm{b}}$ Pearson's chi-squared test, and ${ }^{\mathrm{C}}$ Fisher's exact test.

and overall survival $(P<0.0001)$ than did others (Figures 3(a) and 3(b)). Among patients with carcinoembryonic antigen levels below the threshold, disease-free $(P=0.259)$ and overall survival $(P=0.374)$ was comparable between those with exon 21 mutations and exon 19 deletions (Figures 3(c) and 3(d)). Among patients with carcinoembryonic antigen levels above the threshold, disease-free $(P=0.019)$ and overall survival $(P<$ $0.0001)$ was better in those with exon 21 mutations than in those with exon 19 deletions (Figures 3(e) and $3(\mathrm{f}))$.

3.3. Univariate and Multivariate Analyses for Pathological T1 Lung Adenocarcinoma. Univariate and multivariate Cox regression analysis results for disease-free and overall survival are summarized in Table 4 . In the multivariate analysis, unfavourable disease-free survival was associated with preoperational carcinoembryonic antigen levels 

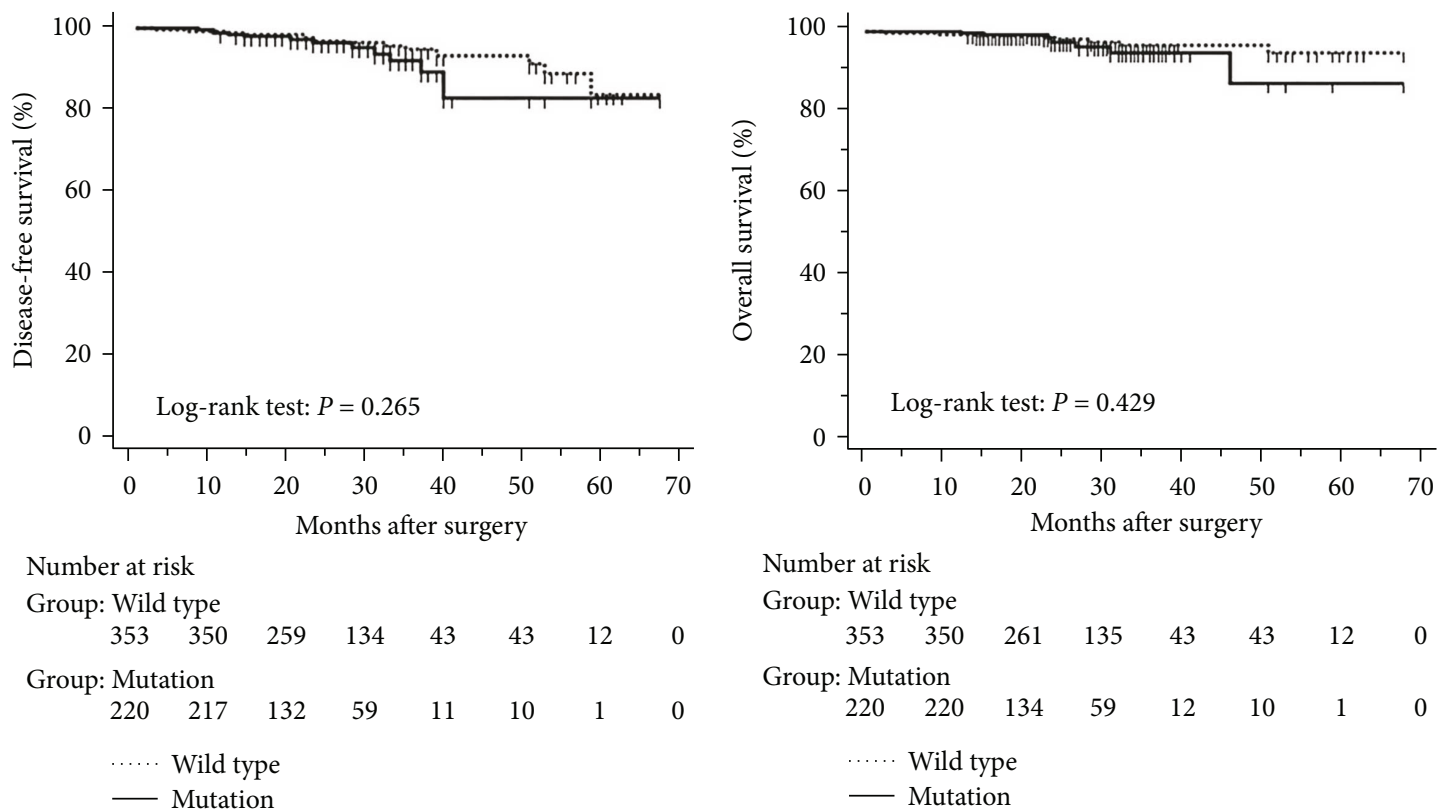

(a)
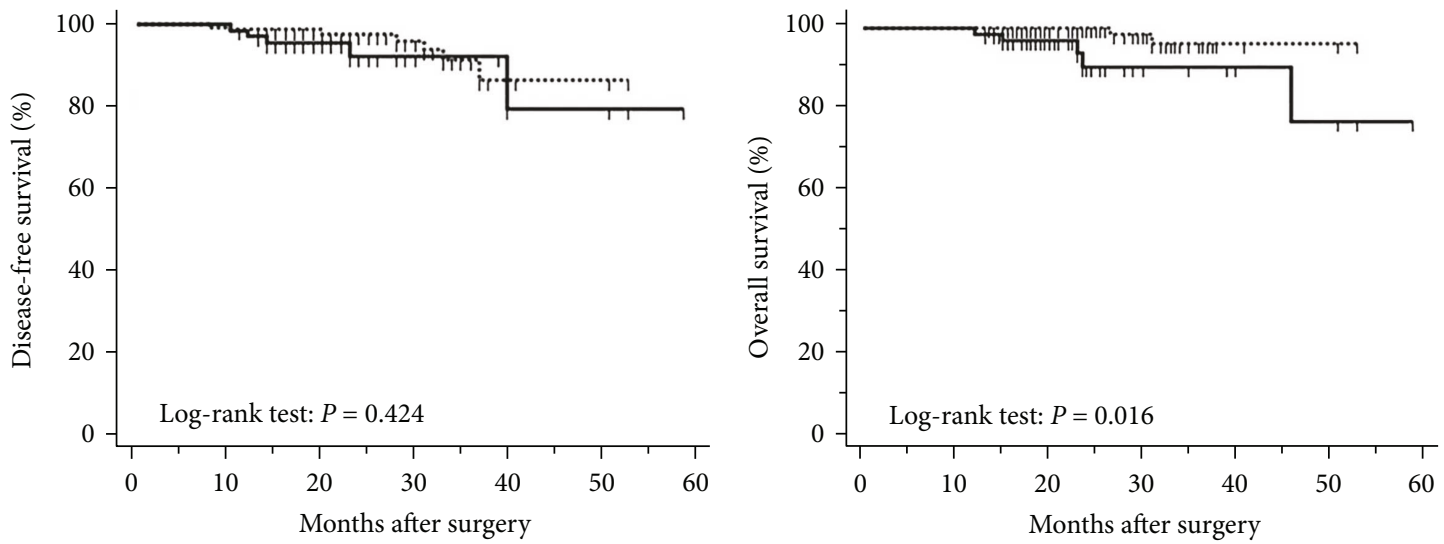

Number at risk

Group: Exon 21

$\begin{array}{llllll}145 \quad 143 & 90 & 43 & 5 & 4 & 0 \\ \text { Group: Exon } 19 & & & & & \\ 64 \quad 63 & 36 & 12 & 5 & 5 & 0 \\ \cdots & \text { Exon } 21 \\ \cdots & & & & & \\ & \text { Exon } 19\end{array}$

(c)

Number at risk

Group: Exon 21

$\begin{array}{llllll}145 \quad 145 & 91 & 43 & 5 & 4 & 0 \\ \text { Group: Exon } 19 & & & & & \\ 64 \quad 64 & 37 & 12 & 6 & 5 & 0 \\ \cdots & \text { Exon } 21 \\ & & & & & \\ \text { - Exon } 19\end{array}$

(d)

FIgURE 2: Kaplan-Meier curves after surgery in 573 lung adenocarcinoma patients with tumours with a maximum diameter of $3 \mathrm{~cm}$ or less. (a) Disease-free and (b) overall survival stratified by presence and absence of EGFR mutations. (c) Disease-free and (d) overall survival stratified by EGFR mutations in exon 19 and exon 21.

above $2.12 \mathrm{ng} / \mathrm{mL}(P=0.022)$, IAC pathology $(P=0.046)$, confirmed lymphatic metastasis $(P<0.001)$, and advanced pathological stage $(P=0.033)$. The exon 19 deletion was an independent predictor of reduced overall survival $(P=0.037)$.

Among patients with carcinoembryonic antigen levels above the threshold (Table 5), exon 19 deletion $(P=0.031)$, large tumour size $(P=0.001)$, IAC pathology $(P=0.012)$, confirmed lymphatic metastasis $(P=0.001)$, and advanced pathological stage $(P=0.029)$ were found by univariate analysis to be unfavourable for disease-free survival. In contrast, large tumour size $(P=0.009)$ and lymphatic metastasis $(P=0.003)$ were predictive of worse overall survival. In the multivariate analysis, none of the clinical features were significantly associated with disease-free survival, although lymphatic metastasis was an independent predictor of reduced overall survival $(P=0.039)$.

3.4. Disease-Free and Overall Survival Excluding Stage 0 Patients. The progression of lung cancer is typically divided 


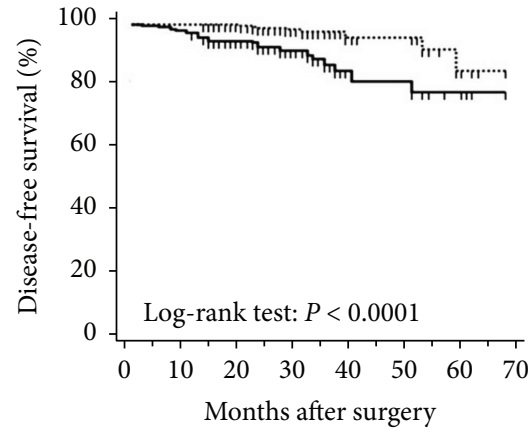

Number at risk

$$
\begin{aligned}
& \text { Group: Low CEA } \\
& \begin{array}{llllllll}
378 & 378 & 262 & 129 & 33 & 32 & 7 & 0
\end{array} \\
& \text { Group: High CEA } \\
& \begin{array}{llllllll}
195 & 189 & 129 & 64 & 21 & 21 & 6 & 0
\end{array} \\
& \text {... } \mathrm{CEA}<2.12 \mathrm{ng} / \mathrm{mL} \\
& \text { - } C E A \geq 2.12 \mathrm{ng} / \mathrm{mL}
\end{aligned}
$$

(a)

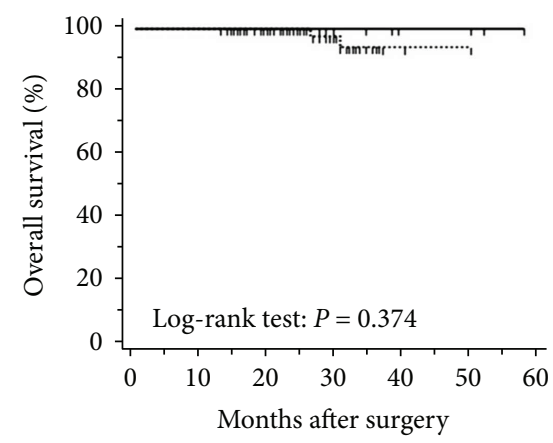

Number at risk

Group: Exon 21

$$
\begin{array}{crrrrrr}
82 & 82 & 50 & 24 & 2 & 1 & 0 \\
\text { Group: Exon } \begin{array}{rrrrrr}
19 \\
42
\end{array} & 42 & 24 & 9 & 5 & 5 & 0 \\
& \\
\text { … } & \text { Exon } 21 & & & & \\
- & \text { Exon } 19
\end{array}
$$

(d)

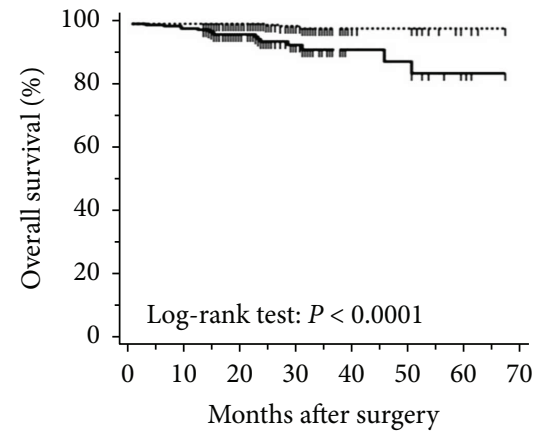

Number at risk

Group: Low CEA

$\begin{array}{llllllll}378 & 378 & 263 & 129 & 33 & 32 & 7 & 0\end{array}$

Group: High CEA

$\begin{array}{llllllll}195 & 192 & 132 & 65 & 22 & 21 & 6 & 0\end{array}$

… $\mathrm{CEA}<2.12 \mathrm{ng} / \mathrm{mL}$

- $\mathrm{CEA} \geq 2.12 \mathrm{ng} / \mathrm{mL}$

(b)

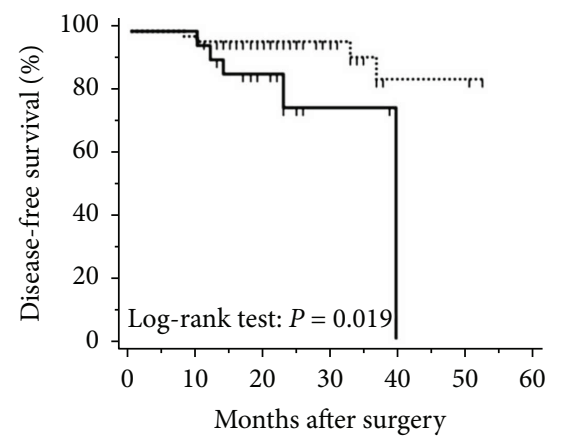

Number at risk

Group: Exon 21

$\begin{array}{lrrrrrr}63 & 61 & 41 & 19 & 3 & 3 & 0 \\ \text { Group: Exon } 19 & & & & & \\ 22 & 21 & 12 & 3 & 0 & 0 & 0 \\ \cdots & & \text { Exon } 21 \\ & & & & & & \\ & \text { Exon } 19\end{array}$

(e)

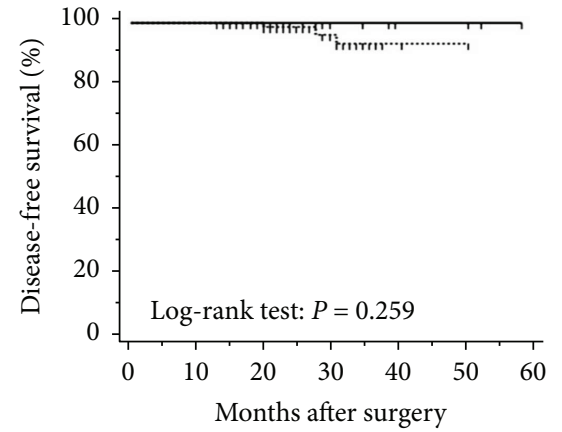

Number at risk

Group: Exon 21

$$
\begin{aligned}
& \begin{array}{lrlllll}
82 & 82 & 49 & 24 & 2 & 1 & 0
\end{array} \\
& \begin{array}{lllllll}
42 & 42 & 24 & 9 & 5 & 5 & 0
\end{array} \\
& \text { … Exon } 21
\end{aligned}
$$

(c)

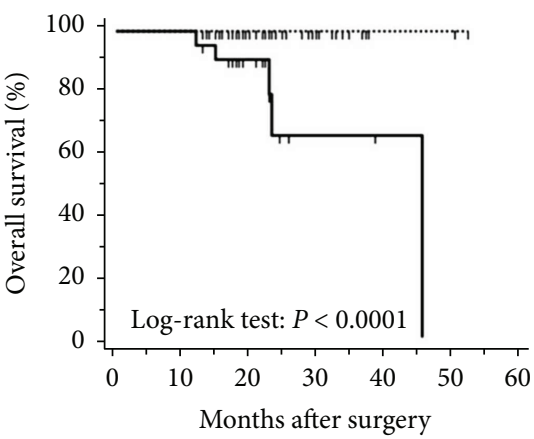

Number at risk

Group: Exon 21

$$
\begin{array}{rrrrrrr}
63 & 63 & 41 & 19 & 3 & 3 & 0 \\
\text { Group: Exon } 19 & & & & & \\
22 & 22 & 13 & 3 & 1 & 0 & 0 \\
\cdots & & & \text { Exon } 21 \\
& - & & & & \\
& \text { Exon } 19
\end{array}
$$

(f)

Figure 3: Kaplan-Meier curves after surgery in lung adenocarcinoma patients with tumours with a maximum diameter of $3 \mathrm{~cm}$ or less. (a) Disease-free and (b) overall survival stratified by preoperational serum carcinoembryonic antigen levels with a cut-off of $2.12 \mathrm{ng} / \mathrm{mL}$. (c) Disease-free and (d) overall survival in patients with carcinoembryonic antigen levels below $2.12 \mathrm{ng} / \mathrm{mL}$ and stratified by mutations in exon 19 and 21. (e) Disease-free and (f) overall survival in patients with carcinoembryonic antigen levels above $2.12 \mathrm{ng} / \mathrm{mL}$ and stratified by mutations in exon 19 and 21 .

into five stages (0-IV); however, the stage 0 lung cancer patients had better prognosis with $100 \%$ disease-free survival and overall survival, and we thus excluded stage 0 patients to analyse the data again [17-19]. The mean follow-up time was 28.0 months, with a median of 25.5 months and range of 2.5-68 months. As shown in Figures 4 and 5 , the results were similar to those for the whole cohort, and patients with exon 21 mutations had significantly extended overall survival than had patients with exon 19 mutations ( $P=0.019$, Figure 4). Additionally, patients with a higher level of serum carcinoembryonic antigen levels showed worse disease-free $(P<0.001)$ and overall survival $(P<0.001)$ than did others, and those with exon 21 mutations had better disease-free $(P=0.025)$ and overall survival $(P<0.001)$ than had those with exon 19 deletions (Figure 5).

\subsection{Univariate and Multivariate Analyses for Pathological T1} Lung Adenocarcinoma. Univariate and multivariate Cox regression analysis results for disease-free and overall survival of patients excluding those in stage 0 are summarized in Table 6 . In the multivariate analysis, unfavourable disease-free survival was correlated with preoperational carcinoembryonic antigen levels above $2.12 \mathrm{ng} / \mathrm{mL}(P=0.031)$, confirmed lymphatic metastasis $(P<0.001)$, and advanced pathological stage $(P=0.012)$. The exon 19 deletion was an independent predictor of reduced overall survival $(P=0.036)$. 
TABLE 4: Univariate and multivariate analysis results of disease-free and overall survival in patients with T1 lung adenocarcinoma.

\begin{tabular}{|c|c|c|c|c|c|c|}
\hline \multirow{2}{*}{ Factor } & \multicolumn{3}{|c|}{ Univariate analysis } & \multicolumn{3}{|c|}{ Multivariate analysis } \\
\hline & HR & $95 \% \mathrm{CI}$ & $P$ & HR & $95 \% \mathrm{CI}$ & $P$ \\
\hline \multicolumn{7}{|l|}{ Disease-free survival } \\
\hline Sex (female versus male) & 2.303 & $1.111-4.775$ & $0.025^{*}$ & 1.268 & $0.583-2.758$ & 0.550 \\
\hline CEA $(<2.12$ versus $\geq 2.12)$ & 4.999 & $2.211-11.302$ & $<0.001^{*}$ & 2.877 & $1.166-7.097$ & $0.022^{*}$ \\
\hline Tumour size (pT1a versus pT1b versus pT1c) & 2.709 & $1.664-4.411$ & $<0.001^{*}$ & 1.454 & $0.786-2.687$ & 0.233 \\
\hline Histology (AIS and MIA versus IAC) & 6.300 & $2.400-16.540$ & $<0.001^{*}$ & 3.204 & $1.023-10.040$ & $0.046^{*}$ \\
\hline Lymphatic metastasis (absent versus present) & 8.901 & $3.934-20.139$ & $<0.001^{*}$ & 11.201 & $2.658-47.209$ & $0.001^{*}$ \\
\hline Pathological stage (stage 0 versus I, II, and IIIA) & 2.128 & $1.430-3.168$ & $<0.001^{*}$ & 0.361 & $0.163-0.800$ & $0.012^{*}$ \\
\hline \multicolumn{7}{|l|}{ Overall survival } \\
\hline Sex (female versus male) & 3.472 & $1.320-9.130$ & $0.012^{*}$ & 2.070 & $0.308-13.899$ & 0.454 \\
\hline Smoking (non versus current/former) & 3.235 & $1.231-8.506$ & $0.017^{*}$ & 1.276 & $0.129-12.663$ & 0.835 \\
\hline CEA $(<2.12$ versus $\geq 2.12)$ & 9.200 & $2.641-32.049$ & $0.001^{*}$ & 2.626 & $0.352-19.583$ & 0.346 \\
\hline EGFR mutation (exon 21 versus exon 19) & 6.170 & $1.162-32.768$ & $0.033^{*}$ & 7.153 & $1.124-45.516$ & $0.037^{*}$ \\
\hline Tumour size (pT1a versus $\mathrm{pT} 1 \mathrm{~b}$ versus $\mathrm{pT} 1 \mathrm{c})$ & 4.056 & $2.046-8.039$ & $<0.001^{*}$ & 5.793 & $0.934-35.944$ & 0.059 \\
\hline Histology (AIS and MIA versus IAC) & 21.184 & $2.187-124.759$ & $0.003^{*}$ & 36416.346 & $0.000-3.671 \mathrm{E} 220$ & 0.967 \\
\hline Lymphatic metastasis (absent versus present) & 9.309 & $3.275-26.457$ & $<0.001^{*}$ & 14.820 & $0.172-1276.425$ & 0.236 \\
\hline Pathological stage (stage 0 versus I, II, and IIIA) & 2.201 & $1.319-3.673$ & $0.003^{*}$ & 0.448 & $0.045-4.468$ & 0.494 \\
\hline
\end{tabular}

HR: hazard ratio; CI: confidence interval; CEA: carcinoembryonic antigen; AIS: adenocarcinoma in situ; MIA: minimally invasive adenocarcinoma; IAC: invasive adenocarcinoma. ${ }^{*} P<0.05$.

TABLE 5: Univariate and multivariate analysis results of disease-free and overall survival in patients with carcinoembryonic antigen $(\mathrm{CEA}) \geq 2.12 \mathrm{IU} / \mathrm{mL}$.

\begin{tabular}{|c|c|c|c|c|c|c|}
\hline \multirow{2}{*}{ Factor } & \multicolumn{3}{|c|}{ Univariate analysis } & \multicolumn{3}{|c|}{ Multivariate analysis } \\
\hline & HR & $95 \% \mathrm{CI}$ & $P$ & HR & $95 \% \mathrm{CI}$ & $P$ \\
\hline \multicolumn{7}{|l|}{ Disease-free survival } \\
\hline EGFR mutation (Ex21 versus Ex19) & 4.281 & $1.138-16.105$ & $0.031^{*}$ & 2.705 & $0.657-11.136$ & 0.168 \\
\hline Tumour size (pT1a versus pT1b versus pT1c) & 2.910 & $1.506-5.623$ & $0.001^{*}$ & 2.813 & $0.551-14.358$ & 0.214 \\
\hline Histology (AIS and MIA versus IAC) & 13.031 & $1.748-97.143$ & $0.012^{*}$ & 76752.907 & $0.000-6.609 \mathrm{E} 256$ & 0.970 \\
\hline Lymphatic metastasis (absent versus present) & 4.811 & $1.926-12.018$ & $0.001^{*}$ & 9.748 & $0.262-362.601$ & 0.217 \\
\hline Pathological stage (stage 0 versus I, II, and IIIA) & 1.664 & $1.054-2.627$ & $0.029^{*}$ & 0.772 & $0.099-6.005$ & 0.804 \\
\hline \multicolumn{7}{|l|}{ Overall survival } \\
\hline Tumour size (pT1a versus $\mathrm{pT} 1 \mathrm{~b}$ versus $\mathrm{pT} 1 \mathrm{c})$ & 2.966 & $1.318-6.674$ & $0.009^{*}$ & 3.204 & $0.989-10.382$ & 0.052 \\
\hline Lymphatic metastasis (absent versus present) & 5.216 & $1.725-15.7737$ & $0.003^{*}$ & 2.444 & $1.044-5.720$ & $0.039^{*}$ \\
\hline
\end{tabular}

HR: hazard ratio; CI: confidence interval; AIS: adenocarcinoma in situ; MIA: minimally invasive adenocarcinoma; IAC: invasive adenocarcinoma. ${ }^{*} P<0.05$.

\section{Discussion}

We surveyed the prognostic value of EGFR mutations in a cohort of 573 patients from East China who underwent surgical resection of pathological T1 lung carcinoma. In particular, we analysed EGFR mutations in patients with preoperational carcinoembryonic antigen levels $\geq 2.12 \mathrm{ng} / \mathrm{mL}$, a key prognostic threshold identified in our previous study [15]. The data suggest that although disease-free and overall survival was comparable between patients with or without EGFR mutations, patients with exon 21 mutations had extended overall survival in comparison with patients with exon 19 deletion. Extended disease-free and overall survival was also observed in patients with exon 21 mutations who had carcinoembryonic antigen levels above the threshold, but not for patients with the same mutations who had carcinoembryonic antigen levels below the threshold. Accordingly, exon 19 deletion was an independent predictor of reduced overall survival.

EGFR mutations have been reported to be more frequent in Chinese and other Asian populations than in Western populations. Indeed, we detected EGFR mutations in $38.4 \%$ of our cohort, which was in line with previous surveys of stage I lung adenocarcinoma [20], but slightly lower than 

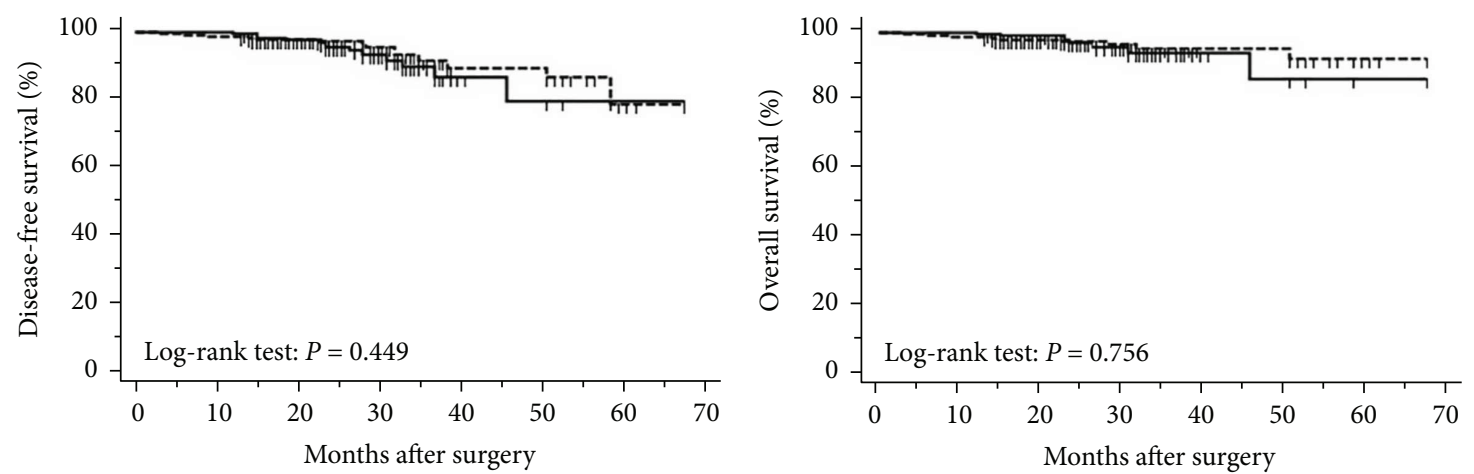

Number at risk

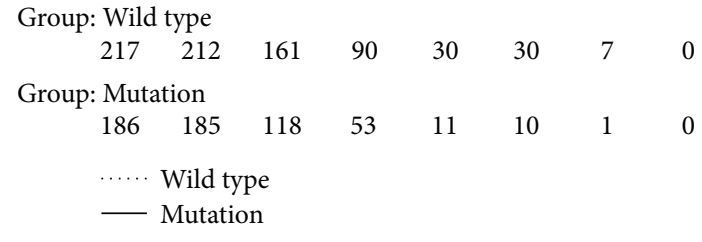

(a)

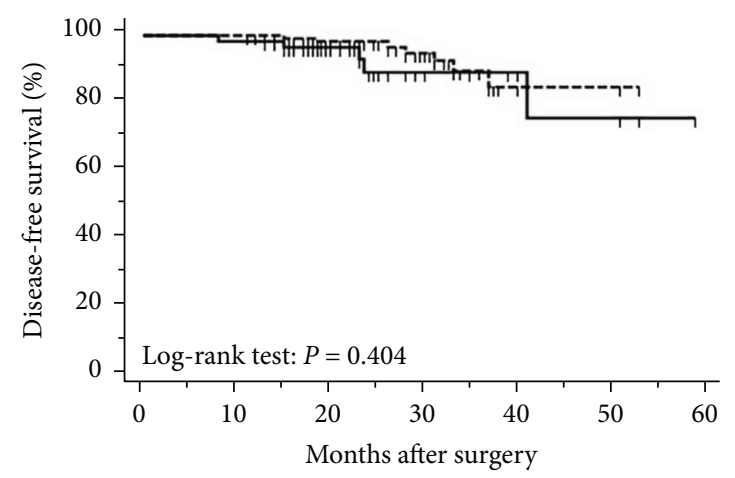

Number at risk Group: Exon 21

$\begin{array}{lclllll}121 \quad 121 & 80 & 39 & 4 & 4 & 0 \\ \text { Group: Exon } 19 & & & & & \\ 58 \quad 57 & 34 & 11 & 6 & 5 & 0 \\ \cdots & \text { Exon } 21 \\ & & & & & \\ - \text { Exon } 19 & & & & \end{array}$

(c)
Number at risk

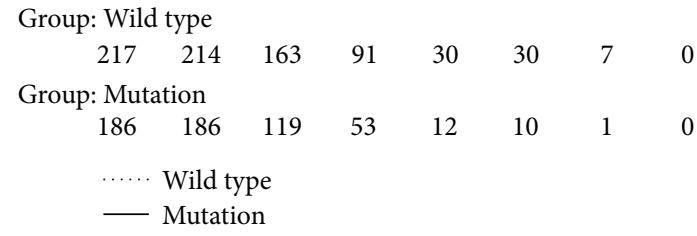

(b)

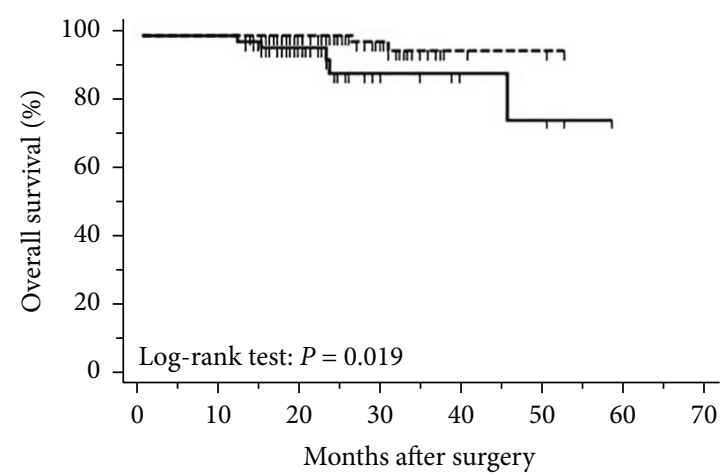

Number at risk Group: Exon 21

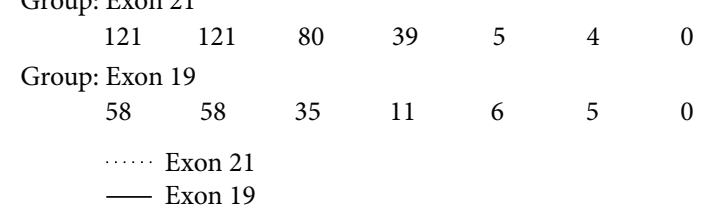

(d)

FIGURE 4: Kaplan-Meier curves after surgery in 403 lung adenocarcinoma patients with tumours with a maximum diameter of $3 \mathrm{~cm}$ or less, excluding those at stage 0 . (a) Disease-free and (b) overall survival stratified by presence and absence of EGFR mutations. (c) Disease-free and (d) overall survival stratified by EGFR mutations in exon 19 and exon 21.

the rate in another report [21]. We attribute this difference to the inclusion in our cohort of a number of patients who had tumours of the adenocarcinoma in situ (AIS) subtype $(29.7 \%)$, in which the frequency of EGFR mutations was reported to be $27.3 \%$ or $23.8 \%$ [22, 23]. In contrast, EGFR mutations in our cohort were most prevalent in patients with acinar forms (62.5\%), and then in patients with lepidic (56.6\%), papillary (54.3\%), minimally invasive adenocarcinoma (MIA) (34.1\%), solid (22.2\%), and AIS $(20.0 \%)$ forms, in line with other studies [23, 24]. In addition, clinical characteristics were comparable between patients with exon 19 and exon 21 mutations, except that lepidic subtypes were more common in the latter than in the former. Conversely, Japanese and Chinese surveys demonstrated that exon 21 mutations were more common than exon 19 mutations in lepidic tumours [11, 25].

On the basis of our previous study, we stratified the patients by serum carcinoembryonic antigen levels [15]. The new data indicate that serum carcinoembryonic antigen levels $\geq 2.12 \mathrm{ng} / \mathrm{mL}$ are associated with EGFR mutations, as previously observed in lung cancer [13, 26, 27]. Strikingly, these mutations were more frequent in nonsmokers with serum carcinoembryonic antigen levels $\geq 2.12 \mathrm{ng} / \mathrm{mL}$, even though the prevalence was comparable between smokers and nonsmokers in the entire study population. Notably, we observed that the prevalence was lower at stage 0 than 


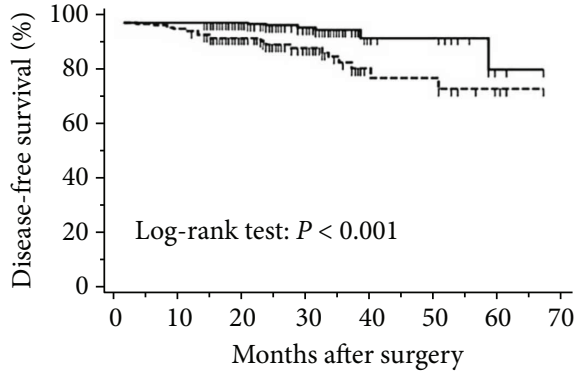

Number at risk

Group: Low CEA

$$
\begin{array}{llllllll}
243 & 243 & 175 & 90 & 23 & 22 & 3 & 0
\end{array}
$$

Group: High CEA

$\begin{array}{llllllll}160 & 154 & 104 & 53 & 18 & 18 & 5 & 0\end{array}$

… CEA $<2.12 \mathrm{ng} / \mathrm{mL}$

$-\mathrm{CEA} \geq 2.12 \mathrm{ng} / \mathrm{mL}$

(a)

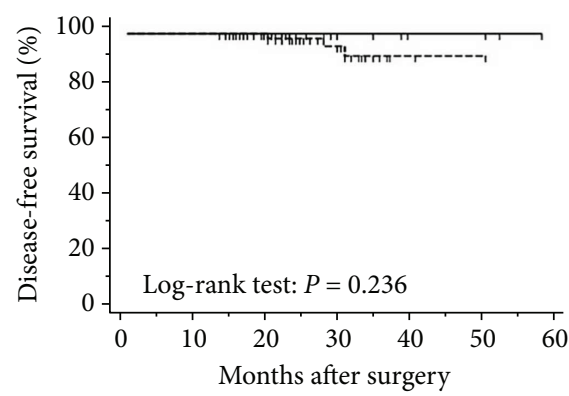

Number at risk

Group: Exon 21

\begin{tabular}{|c|c|c|c|c|c|}
\hline & \multirow[t]{2}{*}{40} & \multirow[t]{2}{*}{21} & \multirow[t]{2}{*}{2} & \multirow[t]{2}{*}{1} \\
\hline & & & & & \\
\hline \multicolumn{2}{|c|}{$\begin{array}{cr}62 & 62 \\
\text { Group: Exon } & 19 \\
36 & 36\end{array}$} & 22 & 8 & & 5 \\
\hline
\end{tabular}

(c)

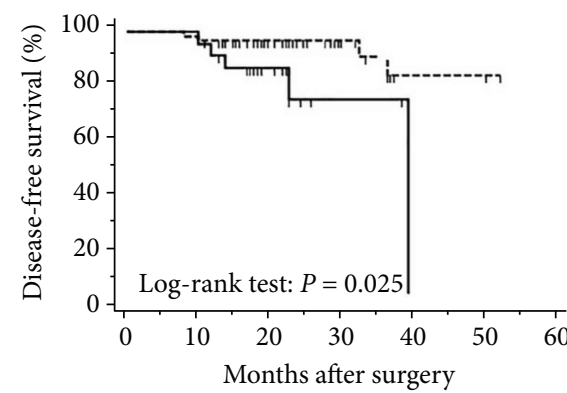

Number at risk

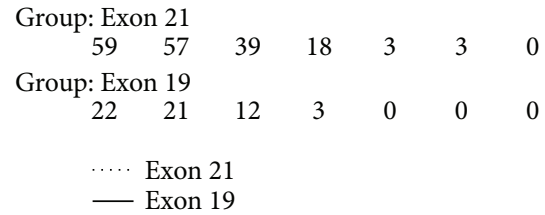

(e)

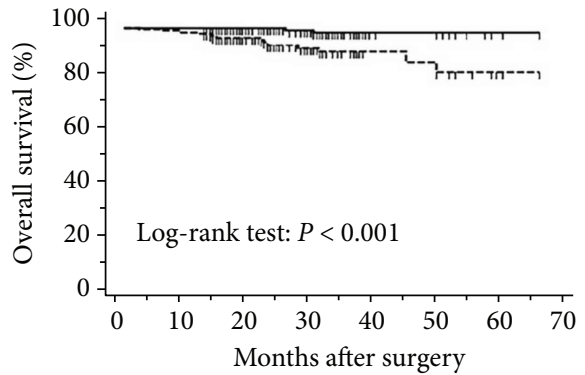

Number at risk

Group: Low CEA

$$
\begin{array}{llllllll}
243 & 243 & 176 & 90 & 23 & 22 & 3 & 0
\end{array}
$$

Group: High CEA

$$
\begin{aligned}
& \begin{array}{llllllll}
160 & 157 & 106 & 54 & 19 & 18 & 5 & 0
\end{array} \\
& \text { … CEA }<2.12 \mathrm{ng} / \mathrm{mL} \\
& \text { - CEA } \geq 2.12 \mathrm{ng} / \mathrm{mL}
\end{aligned}
$$

(b)

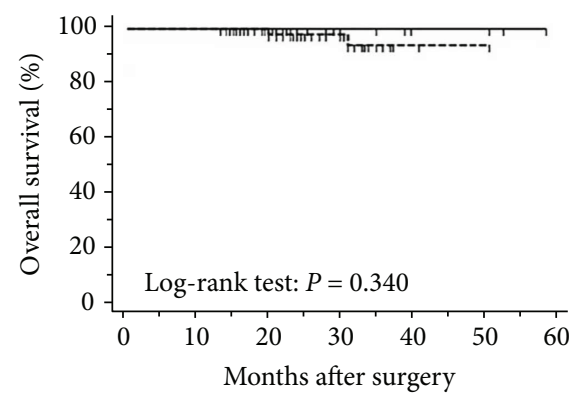

Number at risk

Group: Exon 21

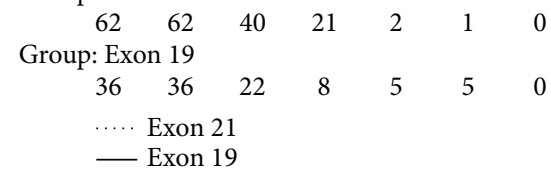

(d)

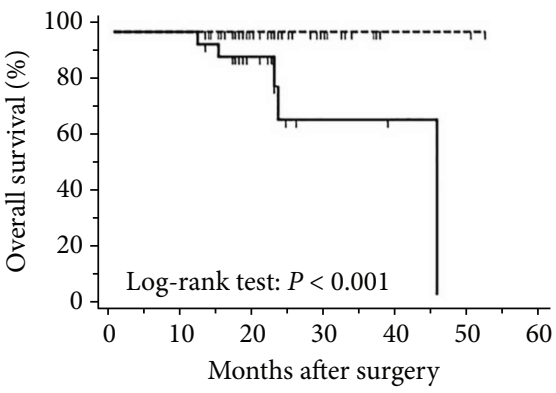

Number at risk

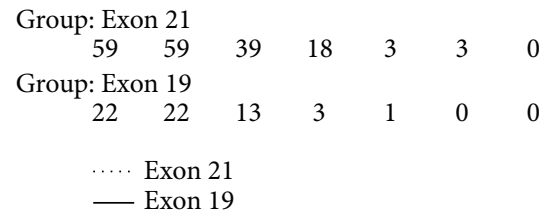

(f)

FIGURE 5: Kaplan-Meier curves after surgery in lung adenocarcinoma patients with tumours with a maximum diameter of $3 \mathrm{~cm}$ or less, excluding those at stage 0 . (a) Disease-free and (b) overall survival stratified by preoperational serum carcinoembryonic antigen levels with a cut-off of $2.12 \mathrm{ng} / \mathrm{mL}$. (c) Disease-free and (d) overall survival in patients with carcinoembryonic antigen levels below $2.12 \mathrm{ng} / \mathrm{mL}$ and stratified by mutations in exon 19 and 21. (e) Disease-free and (f) overall survival in patients with carcinoembryonic antigen levels above $2.12 \mathrm{ng} / \mathrm{mL}$ and stratified by mutations in exon 19 and 21 . 
TABLE 6: Univariate and multivariate analysis results of disease-free and overall survival in patients with T1 lung adenocarcinoma, excluding those at stage 0 .

\begin{tabular}{|c|c|c|c|c|c|c|}
\hline \multirow{2}{*}{ Factor } & \multicolumn{3}{|c|}{ Univariate analysis } & \multicolumn{3}{|c|}{ Multivariate analysis } \\
\hline & HR & $95 \% \mathrm{CI}$ & $P$ & HR & $95 \% \mathrm{CI}$ & $P$ \\
\hline \multicolumn{7}{|l|}{ Disease-free survival } \\
\hline Sex (female versus male) & 2.320 & $1.072-5.023$ & $0.033^{*}$ & 1.377 & $0.601-3.157$ & 0.450 \\
\hline CEA $(<2.12$ versus $\geq 2.12)$ & 5.203 & $2.086-12.977$ & $<0.001^{*}$ & 3.016 & $1.104-8.241$ & $0.031^{*}$ \\
\hline Tumour size (pT1a versus $\mathrm{pT} 1 \mathrm{~b}$ versus $\mathrm{pT} 1 \mathrm{c}$ ) & 2.235 & $1.464-3.412$ & $<0.001^{*}$ & 1.580 & $0.800-3.119$ & 0.188 \\
\hline Histology (MIA versus IAC) & 7.971 & $1.881-33.772$ & $0.005^{*}$ & 1.913 & $0.224-16.317$ & 0.553 \\
\hline Lymphatic metastasis (absent versus present) & 7.183 & $3.117-16.553$ & $<0.001^{*}$ & 19.612 & $3.888-98.923$ & $<0.001^{*}$ \\
\hline Pathological stage (stage I versus II, and IIIA) & 2.054 & $1.263-3.340$ & $0.004^{*}$ & 0.342 & $0.147-0.793$ & $0.012^{*}$ \\
\hline \multicolumn{7}{|l|}{ Overall survival } \\
\hline Sex (female versus male) & 3.332 & $1.208-9.188$ & $0.020^{*}$ & 2.090 & $0.312-14.004$ & 0.447 \\
\hline Smoking (non versus current/former) & 2.817 & $1.047-7.578$ & $0.040^{*}$ & 1.263 & $0.127-12.561$ & 0.842 \\
\hline CEA $(<2.12$ versus $\geq 2.12)$ & 11.244 & $2.552-49.537$ & $0.001^{*}$ & 2.643 & $0.352-19.842$ & 0.345 \\
\hline EGFR mutation (exon 21 versus exon 19) & 5.974 & $1.124-31.746$ & $0.036^{*}$ & 7.221 & $1.133-46.003$ & $0.036^{*}$ \\
\hline Tumour size (pT1a versus $p$ T1b versus $p T 1 c)$ & 3.351 & $1.698-6.613$ & $<0.001^{*}$ & 6.019 & $1.036-34.958$ & $0.046^{*}$ \\
\hline Lymphatic metastasis (absent versus present) & 7.147 & $2.479-20.606$ & $<0.001^{*}$ & 14.645 & $0.170-1258.671$ & 0.238 \\
\hline Pathological stage (stage I versus II, and IIIA) & 1.898 & $1.011-3.564$ & $0.046^{*}$ & 0.449 & $0.045-4.503$ & 0.496 \\
\hline
\end{tabular}

HR: hazard ratio; CI: confidence interval; CEA: carcinoembryonic antigen; AIS: adenocarcinoma in situ; MIA: minimally invasive adenocarcinoma; IAC: invasive adenocarcinoma. ${ }^{*} P<0.05$.

at stages I-III, in contrast to previous surveys [13, 24]. Moreover, we report for the first time that EGFR mutations are less frequent in patients with tumour size pT1a than in patients with tumour size pT1b and pT1c. We note, however, that our cohort included a greater proportion of lung adenocarcinoma at stage 0 and tumour size pTla than had previous cohorts covering stages II to IV. Hence, further prospective surveys are necessary to confirm this result.

Previous surveys of the prognostic value of preoperational serum carcinoembryonic antigen levels have been contradictory, although we note that such surveys use different thresholds [13, 26]. Previously, we determined that levels higher than $2.12 \mathrm{ng} / \mathrm{mL}$ were associated with the prognosis of non-small cell lung cancer, and we now report that patients with levels above this threshold had worse disease-free and overall survival than had patients with levels below this threshold. Indeed, carcinoembryonic antigen levels above $2.12 \mathrm{ng} / \mathrm{mL}$ were an independent predictor of unfavourable prognosis. Similarly, Yang et al. [25] found that levels above $5 \mathrm{ng} / \mathrm{mL}$ were an independent predictor of recurrence-free and overall survival in stage I lung adenocarcinoma. In contrast, in lung adenocarcinoma patients treated with EGFR tyrosine kinase inhibitors and platinum-based doublet chemotherapy, carcinoembryonic antigen levels $>5 \mathrm{ng} / \mathrm{mL}$ were associated with unfavourable prognosis in patients without EGFR mutations, but not in patients with EGFR mutations $[14,28]$. Strikingly, we found that overall survival, but not disease-free survival, was poorer for patients with exon 21 mutations than for patients with exon 19 mutations. This result contradicts findings in patients with advanced unresectable lung adenocarcinoma who were treated with EGFR tyrosine kinase inhibitors but is consistent with a survey by Nishii et al. [29]. In patients with carcinoembryonic antigen levels $\geq 2.12 \mathrm{ng} / \mathrm{mL}$, overall and disease-free survival was also better in patients with exon 21 mutations than in those with exon 19 mutations. However, this relationship was not observed in patients with serum carcinoembryonic antigen levels below $2.12 \mathrm{ng} / \mathrm{mL}$, presumably because the antigen is antiapoptotic. In addition, activation of downstream molecules by EGFR mutants may promote antiapoptotic activity, or mutated EGFR may elicit abundant expression of the antigen $[26,30]$. We also analysed the prognosis of patients excluding those at stage 0 ; however, the results were similar to those obtained for the overall cohort, which confirms the prognostic role of carcinoembryonic antigen and EGFR.

Ultimately, we found that carcinoembryonic antigen levels $\geq 2.12 \mathrm{ng} / \mathrm{mL}$, IAC subtype, lymphatic metastasis, and advanced pathological stage were predictors of worse progression-free survival. Similarly, exon 19 mutations in $E G F R$ were an independent predictor of reduced overall survival, especially in patients with carcinoembryonic antigen levels above $2.12 \mathrm{ng} / \mathrm{mL}$. The mechanism underlying the association of carcinoembryonic antigen with EGFR mutations remains unclear, and molecular studies are needed to investigate the difference in proliferation and survival between tumours with exon 19 and exon 21 mutations.

Our survey is limited by its retrospective nature, inclusion of several stage 0 patients, small sample size, and patient recruitment in a single institution in Eastern China, which may have resulted in selection bias. Nevertheless, the data imply that in pathological T1 lung adenocarcinoma, EGFR mutations are associated with preoperational serum carcinoembryonic levels $\geq 2.12 \mathrm{ng} / \mathrm{mL}$. The data also imply 
that patients with exon 19 deletions in EGFR have less favourable prognosis than had those with exon 21 mutations after curative resection of the lung, especially in patients with carcinoembryonic antigen levels above $\geq 2.12 \mathrm{ng} / \mathrm{mL}$. Thus, carcinoembryonic antigen levels and EGFR genotype should be considered together to assess prognosis in pathological T1 lung adenocarcinoma.

\section{Disclosure}

The funders had no role in study design, data collection or analysis, decision to publish, or preparation of the manuscript.

\section{Conflicts of Interest}

The authors declare that they have no competing interests.

\section{Authors' Contributions}

Wang-Yu Zhu and Hai-Feng Li contributed equally to this work.

\section{Acknowledgments}

This research was supported in part by grants from Natural Science Foundation of Zhejiang Province (China) (Grant no. LQ17H160001) and Medical Bureau of Zhejiang Province (China) (Grant nos. 2015ZDA032 and 2016RCB020) to Wang-Yu Zhu and Han-Bo Le, from Science and Technology Department of Zhejiang Province (China) (Grant no. 2016C37008) to Xiao-Fei $\mathrm{Hu}$, and from the Science and Technology Bureau of Zhoushan to Wang-Yu Zhu (Grant no. 2011C12040).

\section{References}

[1] L. A. Torre, R. L. Siegel, and A. Jemal, "Lung cancer statistics," Advances in Experimental Medicine and Biology, vol. 893, pp. 1-19, 2016.

[2] W. D. Travis, E. Brambilla, and G. J. Riely, "New pathologic classification of lung cancer: relevance for clinical practice and clinical trials," Journal of Clinical Oncology, vol. 31, no. 8, pp. 992-1001, 2013.

[3] J. J. Hung, Y. C. Yeh, W. J. Jeng et al., "Predictive value of the International Association for the Study of Lung Cancer/ American Thoracic Society/European Respiratory Society classification of lung adenocarcinoma in tumor recurrence and patient survival," Journal of Clinical Oncology, vol. 32, no. 22, pp. 2357-2364, 2014

[4] W. Pao and V. A. Miller, "Epidermal growth factor receptor mutations, small-molecule kinase inhibitors, and non-smallcell lung cancer: current knowledge and future directions," Journal of Clinical Oncology, vol. 23, no. 11, pp. 2556-2568, 2005.

[5] W. Pao and J. Chmielecki, "Rational, biologically based treatment of EGFR-mutant non-small-cell lung cancer," Nature Reviews Cancer, vol. 10, no. 11, pp. 760-774, 2010.

[6] C. H. Chiu, T. Y. Chou, C. L. Chiang, and C. M. Tsai, "Should EGFR mutations be tested in advanced lung squamous cell carcinomas to guide frontline treatment?," Cancer Chemotherapy and Pharmacology, vol. 74, no. 4, pp. 661-665, 2014
[7] Y. L. Wu, C. Zhou, C. P. Hu et al., "Afatinib versus cisplatin plus gemcitabine for first-line treatment of Asian patients with advanced non-small-cell lung cancer harbouring EGFR mutations (LUX-Lung 6): an open-label, randomised phase 3 trial," The Lancet Oncology, vol. 15, no. 2, pp. 213-222, 2014.

[8] T. Nishii, T. Yokose, Y. Miyagi et al., "Clinicopathological features and EGFR gene mutation status in elderly patients with resected non-small-cell lung cancer," BMC Cancer, vol. 14, no. 1, p. 610, 2014.

[9] B. Izar, L. Sequist, M. Lee et al., "The impact of EGFR mutation status on outcomes in patients with resected stage I non-small cell lung cancers," The Annals of Thoracic Surgery, vol. 96, no. 3, pp. 962-968, 2013.

[10] A. Yoshizawa, S. Sumiyoshi, M. Sonobe et al., "Validation of the IASLC/ATS/ERS lung adenocarcinoma classification for prognosis and association with EGFR and KRAS gene mutations: analysis of 440 Japanese patients," Journal of Thoracic Oncology, vol. 8, no. 1, pp. 52-61, 2013.

[11] T. Isaka, T. Yokose, H. Ito et al., "Correlations between the EGFR mutation status and clinicopathological features of clinical stage I lung adenocarcinoma," Medicine, vol. 94, no. 42, article e1784, 2015.

[12] Y. Zhang, D. He, W. Fang et al., "The difference of clinical characteristics between patients with exon 19 deletion and those with L858R mutation in nonsmall cell lung cancer," Medicine, vol. 94, no. 44, article e1949, 2015.

[13] Z. Cai, "Relationship between serum carcinoembryonic antigen level and epidermal growth factor receptor mutations with the influence on the prognosis of non-small-cell lung cancer patients," OncoTargets and Therapy, vol. 9, pp. 3873-3878, 2016.

[14] R. Jiang, X. Wang, and K. Li, "Predictive and prognostic value of preoperative serum tumor markers is EGFR mutationspecific in resectable non-small-cell lung cancer," Oncotarget, vol. 7, no. 18, pp. 26823-26836, 2016.

[15] W. Y. Zhu, Y. K. Zhang, Z. D. Chai et al., "Identification of factors for the preoperative prediction of tumour subtype and prognosis in patients with T1 lung adenocarcinoma," Disease Markers, vol. 2016, Article ID 9354680, 10 pages, 2016.

[16] A. Warth, T. Muley, M. Meister et al., "The novel histologic International Association for the Study of Lung Cancer/ American Thoracic Society/European Respiratory Society classification system of lung adenocarcinoma is a stageindependent predictor of survival," Journal of Clinical Oncology, vol. 30, no. 13, pp. 1438-1446, 2012.

[17] R. Rami-Porta, V. Bolejack, J. Crowley et al., “The IASLC lung cancer staging project: proposals for the revisions of the T descriptors in the forthcoming eighth edition of the TNM classification for lung cancer," Journal of Thoracic Oncology, vol. 10, no. 7, pp. 990-1003, 2015.

[18] H. Asamura, K. Chansky, J. Crowley et al., "The international association for the study of lung cancer lung cancer staging project: proposals for the revision of the $\mathrm{N}$ descriptors in the forthcoming 8th edition of the TNM classification for lung cancer," Journal of Thoracic Oncology, vol. 10, no. 12, pp. 1675-1684, 2015.

[19] W. E. E. Eberhardt, A. Mitchell, J. Crowley et al., "The IASLC lung cancer staging project: proposals for the revision of the $\mathrm{M}$ descriptors in the forthcoming eighth edition of the TNM classification of lung cancer," Journal of Thoracic Oncology, vol. 10, no. 11, pp. 1515-1522, 2015. 
[20] T. Ohba, G. Toyokawa, T. Kometani et al., "The mutations of the EGFR and K-ras genes in resected stage I lung adenocarcinoma and their clinical significance," Surgery Today, vol. 44, no. 3, pp. 478-486, 2014.

[21] M. Yotsukura, H. Yasuda, T. Shigenobu et al., "Clinical and pathological characteristics of EGFR mutation in operable early-stage lung adenocarcinoma," Lung Cancer, vol. 109, pp. 45-51, 2017.

[22] H. Nakamura, H. Koizumi, H. Kimura, H. Marushima, H. Saji, and M. Takagi, "Epidermal growth factor receptor mutations in adenocarcinoma in situ and minimally invasive adenocarcinoma detected using mutation-specific monoclonal antibodies," Lung Cancer, vol. 99, pp. 143-147, 2016.

[23] H. Nakamura, H. Saji, T. Shinmyo et al., "Association of IASLC/ATS/ERS histologic subtypes of lung adenocarcinoma with epidermal growth factor receptor mutations in 320 resected cases," Clinical Lung Cancer, vol. 16, no. 3, pp. 209$215,2015$.

[24] F. Lu, S. Li, B. Dong, S. Zhang, C. Lv, and Y. Yang, "Identification of lung adenocarcinoma mutation status based on histologic subtype: retrospective analysis of 269 patients," Thoracic Cancer, vol. 7, no. 1, pp. 17-23, 2016.

[25] Y. Yang, Y. Yang, X. Zhou et al., "EGFR L858R mutation is associated with lung adenocarcinoma patients with dominant ground-glass opacity," Lung Cancer, vol. 87, no. 3, pp. 272277, 2015.

[26] F. Facchinetti, R. Aldigeri, R. Aloe, B. Bortesi, A. Ardizzoni, and M. Tiseo, "CEA serum level as early predictive marker of outcome during EGFR-TKI therapy in advanced NSCLC patients," Tumour Biology, vol. 36, no. 8, pp. 5943-5951, 2015.

[27] Y. Y. Chen, T. W. Huang, W. C. Tsai et al., "Risk factors of postoperative recurrences in patients with clinical stage I NSCLC," World Journal of Surgical Oncology, vol. 12, no. 1, p. 10, 2014.

[28] S. Fang, Z. Wang, J. Guo et al., "Correlation between EGFR mutation status and response to first-line platinum-based chemotherapy in patients with advanced non-small cell lung cancer," OncoTargets and Therapy, vol. 7, pp. 1185-1193, 2014.

[29] T. Nishii, T. Yokose, Y. Miyagi et al., "Prognostic value of EGFR mutations in surgically resected pathological stage I lung adenocarcinoma," Asia-Pacific Journal of Clinical Oncology, vol. 13, no. 5, pp. e204-e211, 2017.

[30] T. Wirth, E. Soeth, F. Czubayko, and H. Juhl, "Inhibition of endogenous carcinoembryonic antigen (CEA) increases the apoptotic rate of colon cancer cells and inhibits metastatic tumor growth," Clinical \& Experimental Metastasis, vol. 19, no. 2, pp. 155-160, 2002. 


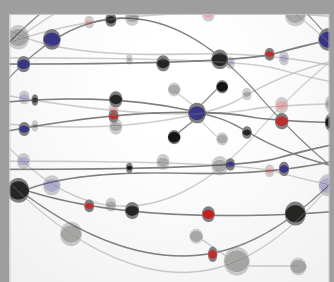

The Scientific World Journal
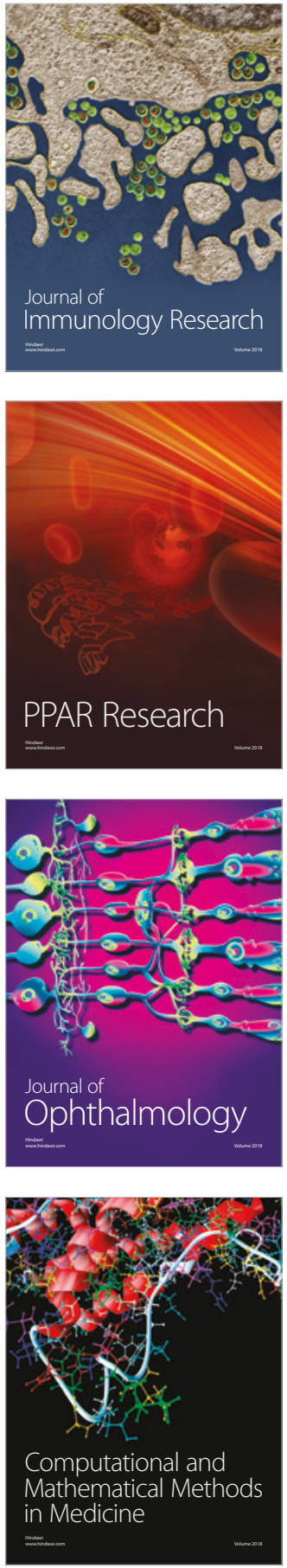

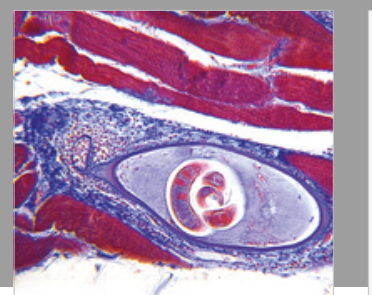

Gastroenterology Research and Practice

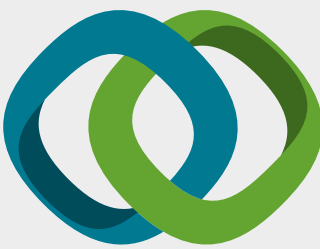

\section{Hindawi}

Submit your manuscripts at

www.hindawi.com
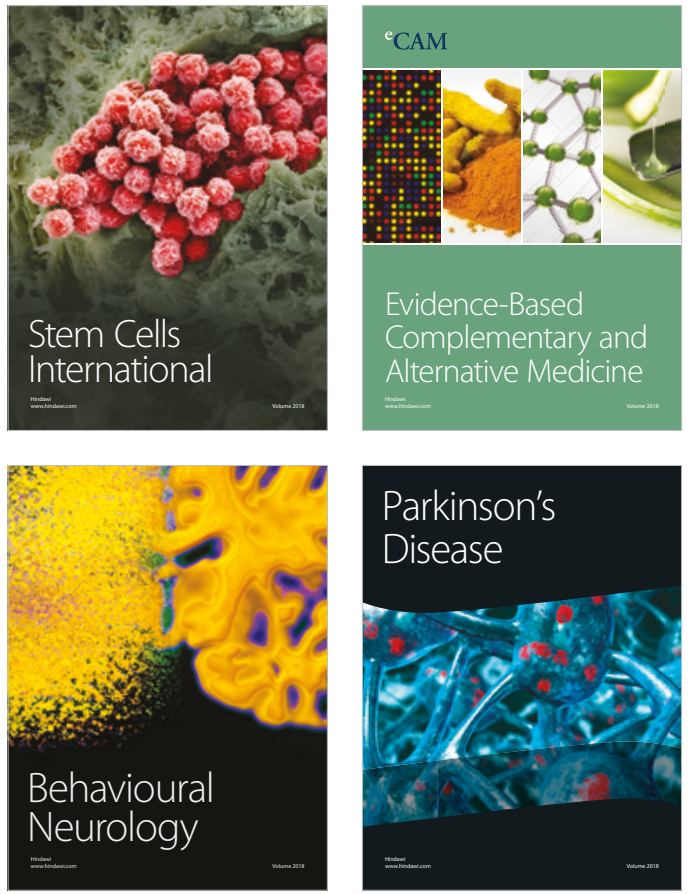

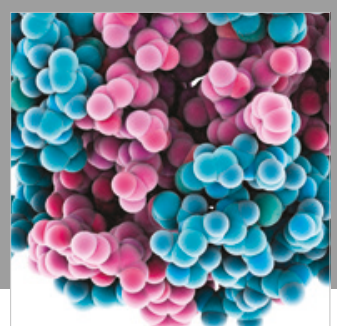

ournal of

Diabetes Research

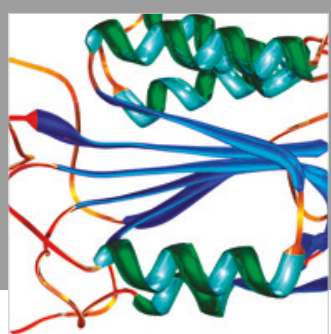

Disease Markers
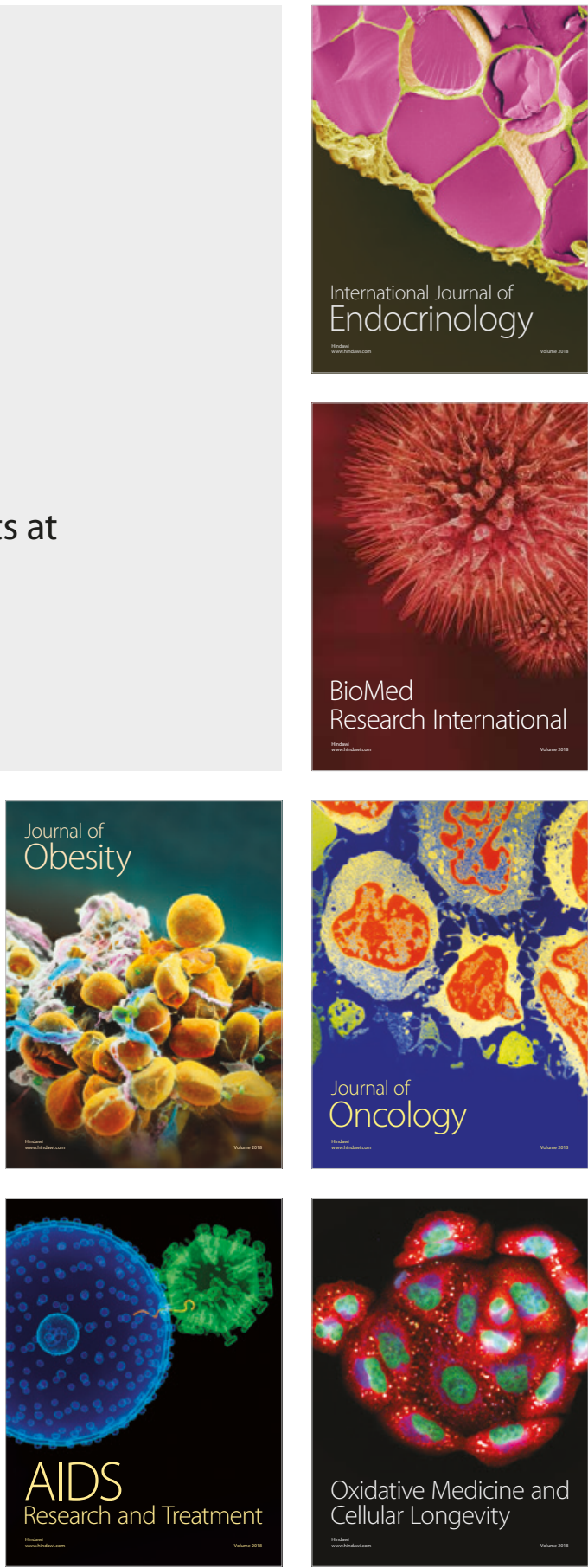